ITEP-TH-01/15

\title{
Quantum Baxter-Belavin R-matrices and multidimensional Lax pairs for Painlevé VI
}

\author{
A. Levin ${ }^{b \sharp} \quad$ M. Olshanetsky $\sharp$ A. Zotov $\diamond \sharp t$ \\ b - NRU HSE, Department of Mathematics, Myasnitskaya str. 20, Moscow, 101000, Russia \\ \#-ITEP, B. Cheremushkinskaya str. 25, Moscow, 117218, Russia \\ घ-MIPT, Inststitutskii per. 9, Dolgoprudny, Moscow region, 141700, Russia \\ $\diamond-$ Steklov Mathematical Institute RAS, Gubkina str. 8, Moscow, 119991, Russia \\ E-mails: alevin@hse.ru, olshanet@itep.ru,zotov@mi.ras.ru
}

\begin{abstract}
The quantum elliptic $R$-matrices of Baxter-Belavin type satisfy the associative YangBaxter equation in $\operatorname{Mat}(N, \mathbb{C})^{\otimes 3}$. The latter can be considered as noncommutative analogue of the Fay identity for the scalar Kronecker function. In this paper we extend the list of $R$-matrix valued analogues of elliptic function identities. In particular, we propose counterparts of the Fay identities in $\operatorname{Mat}(N, \mathbb{C})^{\otimes 2}$. As an application we construct $R$-matrix valued $2 N^{2} \times 2 N^{2}$ Lax pairs for the Painlevé VI equation (in elliptic form) with four free constants using $\mathbb{Z}_{N} \times \mathbb{Z}_{N}$ elliptic $R$-matrix. More precisely, the four free constants case appears for an odd $N$ while even $N$ 's correspond to a single constant.
\end{abstract}




\section{Contents}

1 Introduction and summary $\quad 2$

2 Kronecker double series and Baxter-Belavin $R$-matrix $\quad 9$

3 Derivation of identities $\quad 12$

4 Higher-dimensional elliptic Lax pairs for Painlevé VI 14

\section{Introduction and summary}

In this paper we continue the study of identities for quantum (and classical) $R$-matrices, which are similar to the elliptic functions identities for scalar elliptic functions [13, 8]. More concretely, we prove the Fay identities in $\operatorname{Mat}(N, \mathbb{C})^{\otimes 2}$. It allows us to construct multidimensional Lax pairs for the Painlevé VI equation with the $R$-matrices as matrix elements.

We start with the list of properties and identities for elliptic functions, and then give their $R$-matrix version. Most of the properties are known from [2, 4], [14], [3, 15], [13] and [8].

Consider the following functions:

$$
\begin{gathered}
\phi(z, u)=\frac{\vartheta^{\prime}(0) \vartheta(z+u)}{\vartheta(z) \vartheta(u)}, \\
E_{1}(z)=\frac{\vartheta^{\prime}(z)}{\vartheta(z)}, \quad E_{2}(z)=-\partial_{z} E_{1}(z)=\wp(z)-\frac{1}{3} \frac{\vartheta^{\prime \prime \prime}(0)}{\vartheta^{\prime}(0)},
\end{gathered}
$$

where $\vartheta(z)$ is the odd Riemann theta-function

$$
\vartheta(z)=\vartheta(z \mid \tau)=\sum_{k \in \mathbb{Z}} \exp \left(\pi \imath \tau\left(k+\frac{1}{2}\right)^{2}+2 \pi \imath\left(z+\frac{1}{2}\right)\left(k+\frac{1}{2}\right)\right)
$$

and $\wp(z)$ is the Weierstrass $\wp$-function.

Following [16] the function (1.1) is referred to as the Kronecker function, and (1.2) are called the (first and the second) Eisenstein functions.

The Kronecker function can be considered as a section of the Poincaré bundle $\mathcal{P}$ over $\Sigma_{\tau} \times \Sigma_{\tau}^{\prime}$. Here $\Sigma_{\tau}$ is the elliptic curve

$$
\Sigma_{\tau}=\mathbb{C} /(\mathbb{Z}+\tau \mathbb{Z}), \quad \Im m \tau>0
$$

$\Sigma_{\tau}^{\prime}$ - is its Jacobian $\left(\Sigma_{\tau}^{\prime} \sim \Sigma_{\tau}\right)$. The Poincaré bundle $\mathcal{P}$ is a line bundle over $\Sigma_{\tau} \times \Sigma_{\tau}^{\prime}$ 


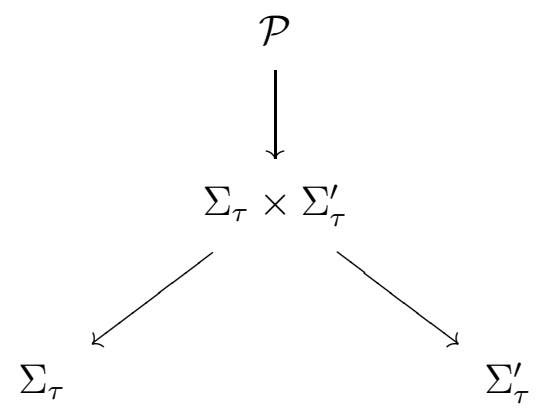

specialized by (1.6), (1.7), (1.10) and (1.11).

The properties of theta-function (1.3) (including Riemann identities, see [11]) provides the following set of properties and relations for the functions (1.1)-(1.2):

- Arguments symmetry:

$$
\phi(z, u)=\phi(u, z), \quad z \in \Sigma_{\tau}, u \in \Sigma_{\tau}^{\prime},
$$

- Local expansion:

$$
\phi(z, u)=\frac{1}{z}+E_{1}(u)+\frac{z}{2}\left(E_{1}^{2}(u)-\wp(u)\right)+O\left(z^{2}\right),
$$

- Residues:

$$
\operatorname{Res}_{z=0} \phi(z, u)=\operatorname{Res}_{u=0} \phi(z, u)=\operatorname{Res}_{z=0} E_{1}(z)=1
$$

- Parity:

$$
\phi(-z,-u)=-\phi(z, u), \quad E_{1}(-z)=-E_{1}(z), \quad E_{2}(-z)=E_{2}(z)
$$

- (Quasi)periodicity properties:

$$
\begin{gathered}
\phi(z+1, u)=\phi(z, u), \quad E_{1}(z+1)=E_{1}(z), \quad E_{2}(z+1)=E_{2}(z), \\
\phi(z+\tau, u)=e^{-2 \pi \imath u} \phi(z, u), \quad E_{1}(z+\tau)=E_{1}(z)-2 \pi \imath, \quad E_{2}(z+\tau)=E_{2}(z),
\end{gathered}
$$

- Heat equation:

$$
2 \pi \imath \partial_{\tau} \phi(z, u)=\partial_{z} \partial_{u} \phi(z, u)
$$

- Derivatives:

$$
\begin{aligned}
& \partial_{u} \phi(z, u)=\phi(z, u)\left(E_{1}(z+u)-E_{1}(u)\right), \\
& \partial_{z} \phi(z, u)=\phi(z, u)\left(E_{1}(z+u)-E_{1}(z)\right),
\end{aligned}
$$

- Fay (trisecant) identity [6]:

$$
\phi(x, u) \phi(y, w)=\phi(x-y, u) \phi(y, u+w)+\phi(y-x, w) \phi(x, u+w),
$$


- Degenerated Fay identities:

$$
\phi(x, z) \phi(x, w)=\phi(x, z+w)\left(E_{1}(x)+E_{1}(z)+E_{1}(w)-E_{1}(x+z+w)\right),
$$

or

$$
\begin{gathered}
\phi(x, z) \phi(y, z)=\phi(x+y, z)\left(E_{1}(x)+E_{1}(y)+E_{1}(z)-E_{1}(x+y+z)\right), \\
\phi(x, z) \phi(x,-z)=E_{2}(x)-E_{2}(z)=\wp(x)-\wp(z) .
\end{gathered}
$$

- Geometric interpretation: The Kronecker function $\phi(z, u)$ is a section of the Poincaré bundle $\mathcal{P}$. It is a line bundle over $\Sigma_{\tau} \times \Sigma_{\tau}$, defined by the conditions (1.6), (1.7), (1.10), (1.11).

- Green function: The Kronecker function is the Green function for the operator $\bar{\partial}$ in the space of one forms $\mathcal{A}^{(1,0)}\left(\Sigma_{\tau}\right)$ with the boundary conditions (1.10) and (1.11):

$$
\bar{\partial} \phi(z, u)=\delta^{2}(z, \bar{z}) .
$$

Quantum $R$-matrices. Consider $\mathbb{Z}_{N} \times \mathbb{Z}_{N}$ (Baxter-Belavin's) elliptic $R$-matrix [2, 4] in the fundamental representation (see also [14]). It is defined via the finite-dimensional representation of the Heisenberg group:

$$
\begin{aligned}
Q, \Lambda \in \operatorname{Mat}(N, \mathbb{C}): \quad Q_{k l}=\delta_{k l} \exp \left(\frac{2 \pi \imath}{N} k\right), \quad \Lambda_{k l}=\delta_{k-l+1=0 \bmod N}, \quad k, l=1, \ldots, N, \\
\\
\exp \left(2 \pi \imath \frac{\gamma_{1} \gamma_{2}}{N}\right) Q^{\gamma_{1}} \Lambda^{\gamma_{2}}=\Lambda^{\gamma_{2}} Q^{\gamma_{1}}, \quad \gamma_{1}, \gamma_{2} \in \mathbb{Z} .
\end{aligned}
$$

Introduce the sin-algebra basis in $\operatorname{Mat}(N, \mathbb{C})$ :

$$
T_{\gamma}:=T_{\gamma_{1} \gamma_{2}}=\exp \left(\pi \imath \frac{\gamma_{1} \gamma_{2}}{N}\right) Q^{\gamma_{1}} \Lambda^{\gamma_{2}}, \quad \gamma_{1}, \gamma_{2}=0, \ldots, N-1 .
$$

The same definition is used for any $\gamma \in \mathbb{Z}^{\times 2}$. Then

$$
T_{\alpha} T_{\beta}=\kappa_{\alpha, \beta} T_{\alpha+\beta}, \quad \kappa_{a, b}=\exp \left(\frac{\pi \imath}{N}\left(\beta_{1} \alpha_{2}-\beta_{2} \alpha_{1}\right)\right),
$$

where $\alpha+\beta=\left(\alpha_{1}+\beta_{1}, \alpha_{2}+\beta_{2}\right)$. The $R$-matrix is defined as

$$
R_{12}^{\hbar}(u)=\sum_{\alpha \in \mathbb{Z}_{N} \times \mathbb{Z}_{N}} \varphi_{\alpha}\left(u, \omega_{\alpha}+\hbar\right) T_{\alpha} \otimes T_{-\alpha} \in \operatorname{Mat}(N, \mathbb{C})^{\otimes 2},
$$

where1

$$
\varphi_{\alpha}\left(u, \omega_{\alpha}+\hbar\right)=\exp \left(2 \pi \imath u \partial_{\tau} \omega_{\alpha}\right) \phi\left(u, \omega_{\alpha}+\hbar\right), \quad \omega_{\alpha}=\frac{\alpha_{1}+\alpha_{2} \tau}{N} .
$$

The $\mathbb{Z}_{N} \times \mathbb{Z}_{N}$ symmetry means that for $g=Q, \Lambda$

$$
(g \otimes g) R_{12}^{\hbar}(u)\left(g^{-1} \otimes g^{-1}\right)=R_{12}^{\hbar}(u) .
$$

\footnotetext{
${ }^{1}$ Here $\partial_{\tau} \omega_{\alpha}=\alpha_{2} / N$
} 
For $N=1$ the $R$-matrix (1.24) is the scalar Kronecker function $\phi(\hbar, u)(1.1)$. Notice that (1.24) is normalized in such a way that the unitarity condition acquires the form:

$$
R_{12}^{\hbar}(u) R_{21}^{\hbar}(-u)=N^{2} \phi(N \hbar, u) \phi(N \hbar,-u) 1 \otimes 1=N^{2}(\wp(N \hbar)-\wp(u)) 1 \otimes 1 .
$$

The latter can be considered as analogue of (1.18). Here $R_{21}(z)=P_{12} R_{12}(z) P_{12}$, where

$$
P_{12}=\frac{1}{N} \sum_{\alpha} T_{\alpha} \otimes T_{-\alpha}=\sum_{i, j=1}^{N} E_{i j} \otimes E_{j i}, \quad\left(E_{i j}\right)_{k l}=\delta_{i k} \delta_{j l}
$$

is the permutation operator. We also use notation $R_{a b}^{\hbar}(z)$ which differs from (1.27) by $T_{\alpha}^{a} \otimes T_{-\alpha}^{b}=$ $1 \otimes \ldots 1 \otimes T_{\alpha} \otimes 1 \ldots 1 \otimes T_{-\alpha} \otimes 1 \ldots \otimes 1$ instead of $T_{\alpha} \otimes T_{-\alpha}$ (i.e. $T_{\alpha}$ and $T_{-\alpha}$ are in the $a$-th and $b$-th components). The number of components in the tensor product is an integer $\tilde{N}$. It means that $R_{a b}^{\hbar}$ is considered as an element of $\operatorname{Mat}(N, \mathbb{C})^{\otimes \tilde{N}}$, i.e. $N^{\tilde{N}} \times N^{\tilde{N}}$ matrix.

The properties and identities (1.8)-(1.17) have the following analogues for $R$-matrices:

- Arguments symmetry:

$$
R_{12}^{\hbar}(z)=R_{12}^{\frac{z}{N}}(N \hbar) P_{12}
$$

- Local expansion in $\hbar$ is the classical limit:

$$
R_{12}^{\hbar}(z)=\hbar^{-1} 1 \otimes 1+r_{12}(z)+\hbar m_{12}(z)+O\left(\hbar^{2}\right)
$$

where $r_{12}(z)$ is the classical (Belavin-Drinfeld [4]) $r$-matrix:

$$
r_{12}(z)=E_{1}(z) 1 \otimes 1+\sum_{\alpha \neq 0} \varphi_{\alpha}(z) T_{\alpha} \otimes T_{-\alpha}
$$

and

$$
m_{12}(z)=\frac{E_{1}^{2}(z)-\wp(z)}{2} 1 \otimes 1+\left.\sum_{\alpha \neq 0} \exp \left(2 \pi \imath z \partial_{\tau} \omega_{\alpha}\right) \partial_{u} \phi(z, u)\right|_{u=\omega_{\alpha}} T_{\alpha} \otimes T_{-\alpha}
$$

Similarly to (1.7) we have:

$$
r_{12}^{2}(z)-2 m_{12}(z)=1 \otimes 1 N^{2} \wp(z)
$$

i.e. the quantum $R$-matrix is a matrix analogue of the Kronecker function (1.1) while the classical one is the analogue of the first Eisenstein function (1.2).

Expansion with respect to $z$ (near $z=0$ ) is as follows:

$$
R_{12}^{\hbar}(z)=\frac{N P_{12}}{z}+R_{12}^{\hbar,(0)}+O(z)
$$

where 2

$$
R_{12}^{\hbar,(0)}=\sum_{\alpha} T_{\alpha} \otimes T_{-\alpha}\left(E_{1}\left(\hbar+\omega_{\alpha}\right)+2 \pi \imath \partial_{\tau} \omega_{\alpha}\right)
$$

\footnotetext{
${ }^{2} R_{12}^{\hbar,(0)}$ appears as a part of the inverse inertia tensor for relativistic tops 9 .
} 
- Residues

$$
\operatorname{Res}_{\hbar=0} R_{12}^{\hbar}(z)=1 \otimes 1, \quad \operatorname{Res}_{z=0} R_{12}^{\hbar}(z)=\operatorname{Res}_{z=0} r_{12}(z)=N P_{12},
$$

- Parity:

$$
R_{12}^{\hbar}(z)=-R_{21}^{-\hbar}(-z), \quad r_{12}(z)=-r_{21}(-z), \quad m_{12}(z)=m_{21}(-z) .
$$

The $R$-matrix analogue of $E_{2}(u)=E_{2}(-u)$ (1.2) appears as $F_{12}^{0}(u)=-\partial_{u} r_{12}(u)$ (It is natural because $r_{12}(u)$ is the analogue of $\left.E_{1}(u)\right)$. The classical $r$-matrix is odd. Hence $F_{12}^{0}(u)$ is even matrix function. The same answer follows from the local expansions (1.7), (1.30): $E_{2}(u)=-\left.\partial_{u} \phi(z, u)\right|_{z=0}$, then $-\left.\partial_{u} R_{12}^{z}(u)\right|_{z=0}=-\partial_{u} r_{12}(u)$.

- (Quasi)periodicity properties:

$$
\begin{gathered}
R_{12}^{\hbar}\left(z+N \omega_{\gamma}\right)=\exp \left(-2 \pi \imath N \hbar \partial_{\tau} \omega_{\gamma}\right)\left(T_{\gamma}^{-1} \otimes 1\right) R_{12}^{\hbar}(z)\left(T_{\gamma} \otimes 1\right), \\
R_{12}^{\hbar+\omega_{\gamma}}(z)=\exp \left(-2 \pi \imath z \partial_{\tau} \omega_{\gamma}\right)\left(T_{\gamma}^{-1} \otimes 1\right) R_{12}^{\hbar}(z)\left(1 \otimes T_{\gamma}\right) .
\end{gathered}
$$

In particular,

$$
\begin{gathered}
R_{12}^{\hbar}(z+1)=\left(Q^{-1} \otimes 1\right) R_{12}^{\hbar}(z)(Q \otimes 1), \\
R_{12}^{\hbar}(z+\tau)=\exp (-2 \pi \imath \hbar)\left(\Lambda^{-1} \otimes 1\right) R_{12}^{\hbar}(z)(\Lambda \otimes 1), \\
R_{12}^{\hbar+1}(z)=R_{12}^{\hbar}(z), \quad R_{12}^{\hbar+\tau}(z)=\exp (-2 \pi \imath z) R_{12}^{\hbar}(z), \\
r_{12}(z+1)=\left(Q^{-1} \otimes 1\right) r_{12}(z)(Q \otimes 1), \\
r_{12}(z+\tau)=\left(\Lambda^{-1} \otimes 1\right) r_{12}(z)(\Lambda \otimes 1)-2 \pi \imath 1 \otimes 1 .
\end{gathered}
$$

Let us also rewrite (1.39) as follows:

$$
\begin{gathered}
R_{a b}^{\hbar+1 / N}\left(z_{a}-z_{b}\right)=Q_{a}^{-1} R_{a b}^{\hbar}\left(z_{a}-z_{b}\right) Q_{b}, \\
R_{a b}^{\hbar+\tau / N}\left(z_{a}-z_{b}\right)=\exp \left(-2 \pi \imath \frac{z_{a}-z_{b}}{N}\right) \Lambda_{a}^{-1} R_{a b}^{\hbar}\left(z_{a}-z_{b}\right) \Lambda_{b} .
\end{gathered}
$$

Recall now the $R$-matrix valued Lax matrix for $\mathrm{g}_{\tilde{N}}$ Calogero-Moser model [8]:

$$
\mathcal{L}(\hbar)=\sum_{a, b=1}^{\tilde{N}} \tilde{\mathrm{E}}_{a b} \otimes \mathcal{L}_{a b}(\hbar), \quad \mathcal{L}_{a b}(\hbar)=\delta_{a b} p_{a} 1_{a} \otimes 1_{b}+\nu\left(1-\delta_{a b}\right) R_{a b}^{\hbar}\left(z_{a}-z_{b}\right) .
$$

where $\tilde{\mathrm{E}}_{a b}$ is the standard basis of $\mathrm{gl}_{\tilde{N}}:\left(\tilde{\mathrm{E}}_{a b}\right)_{c d}=\delta_{a c} \delta_{b d}, a, b, c, d=1 \ldots \tilde{N}$. Then it follows from (1.43)-(1.44) that

$$
\begin{gathered}
\mathcal{L}(\hbar+1 / N)=\mathbf{Q}^{-1} \mathcal{L}(\hbar) \mathbf{Q}, \\
\mathcal{L}(\hbar+\tau / N)=\exp (-\mathbf{Z} / N) \boldsymbol{\Lambda}^{-1} \mathcal{L}(\hbar) \boldsymbol{\Lambda} \exp (\mathbf{Z} / N),
\end{gathered}
$$

where

$$
\mathbf{Q}=\bigoplus_{a=1}^{\tilde{N}} Q_{a}, \quad \mathbf{\Lambda}=\bigoplus_{a=1}^{\tilde{N}} \Lambda_{a}, \quad \mathbf{Z}=\bigoplus_{a=1}^{\tilde{N}} z_{a} 1_{a}
$$

are block diagonal matrices. The number of blocks is $\tilde{N} \times \tilde{N}$, the size of a block is $N^{\tilde{N}} \times N^{\tilde{N}}$. 
- Heat equation:

$$
2 \pi \imath \partial_{\tau} R_{12}^{\hbar}(z)=\partial_{z} \partial_{\hbar} R_{12}^{\hbar}(z) .
$$

- Derivatives 3 :

$$
\begin{gathered}
\partial_{\hbar} R_{12}^{\hbar}(z)=\frac{1}{2}\left(r_{12}(z+N \hbar) R_{12}^{\hbar}(z)+R_{12}^{\hbar}(z) r_{12}(z-N \hbar)\right) \\
+\frac{N}{2}\left(E_{1}(z+N \hbar)-E_{1}(z-N \hbar)-2 E_{1}(N \hbar)\right) R_{12}^{\hbar}(z), \\
\partial_{z} R_{12}^{\hbar}(z)=\frac{1}{2 N}\left(r_{12}(z+N \hbar) R_{12}^{\hbar}(z)-R_{12}^{\hbar}(z) r_{12}(z-N \hbar)\right) \\
+\frac{1}{2}\left(E_{1}(z+N \hbar)+E_{1}(z-N \hbar)-2 E_{1}(z)\right) R_{12}^{\hbar}(z) .
\end{gathered}
$$

- The Fay identity in $\operatorname{Mat}(N, \mathbb{C})^{\otimes 3}[1,13$, 8]:

$$
R_{a b}^{\hbar} R_{b c}^{\hbar^{\prime}}=R_{a c}^{\hbar^{\prime}} R_{a b}^{\hbar-\hbar^{\prime}}+R_{b c}^{\hbar^{\prime}-\hbar} R_{a c}^{\hbar}, \quad R_{a b}^{\hbar}=R_{a b}^{\hbar}\left(z_{a}-z_{b}\right) .
$$

Both parts of the identity are elements of $\operatorname{Mat}(N, \mathbb{C})^{\otimes 3}$. It was used in $[8]$ for constructing higher-dimensional Lax pairs for Calogero-Moser models. Here we will prove another analogue of $(1.15)-$ in $\operatorname{Mat}(N, \mathbb{C})^{\otimes 2}$.

- The Fay identity in $\operatorname{Mat}(N, \mathbb{C})^{\otimes 2}$ :

$$
\begin{gathered}
R_{12}^{\hbar}(z) R_{21}^{\hbar^{\prime}}(-w)= \\
N \phi\left(N \hbar^{\prime}, \frac{z-w}{N}+\hbar^{\prime}-\hbar\right) R_{12}^{\hbar-\hbar^{\prime}}\left(z+N \hbar^{\prime}\right)-N \phi\left(N \hbar, \frac{z-w}{N}+\hbar^{\prime}-\hbar\right) R_{12}^{\hbar-\hbar^{\prime}}(w+N \hbar) \\
+N \phi\left(-w, \frac{z-w}{N}+\hbar^{\prime}-\hbar\right) R_{12}^{\frac{z-w}{N}}(w+N \hbar)-N \phi\left(-z, \frac{z-w}{N}+\hbar^{\prime}-\hbar\right) R_{12}^{\frac{z-w}{N}}\left(z+N \hbar^{\prime}\right) .
\end{gathered}
$$

The scalar analogue of this identity is obtained as follows: apply (1.15) (with $x=\hbar$, $\left.y=\hbar^{\prime}\right)$ to $\phi(\hbar, z) \phi\left(\hbar^{\prime},-w\right)$, and then apply (1.15) once again to the obtained r.h.s.. Then we get the scalar analogue of r.h.s. of (1.52).

- Degenerated Fay identities in $\operatorname{Mat}(N, \mathbb{C})^{\otimes 3}$ (1.51):

$$
\begin{gathered}
R_{a b}^{\hbar} R_{b c}^{\hbar}=R_{a c}^{\hbar} r_{a b}+r_{b c} R_{a c}^{\hbar}-\partial_{\hbar} R_{a c}^{\hbar}, \\
R_{a b}^{\hbar}(z) R_{b c}^{\hbar^{\prime}}(-z)=R_{a c}^{\hbar^{\prime},(0)} R_{a b}^{\hbar-\hbar^{\prime}}(z)+R_{b c}^{\hbar^{\prime}-\hbar}(-z) R_{a c}^{\hbar,(0)}+N F_{b c}^{\hbar^{\prime}-\hbar}(-z) P_{a c},
\end{gathered}
$$

where $F_{a b}^{\hbar}(u)=\partial_{u} R_{a b}^{\hbar}(u)$ and $R_{a b}^{\hbar,(0)}$ is from (1.34) - (1.35).

\footnotetext{
${ }^{3}$ The identities for derivatives of $R$-matrix with respect to the Planck constant and spectral parameter were found in [3] and [15] respectively. Authors of [3, 15] used different normalization of the $R$-matrix.
} 
- Degenerated Fay identities in $\operatorname{Mat}(N, \mathbb{C})^{\otimes 2}$ (1.52):

$$
\begin{gathered}
R_{12}^{\hbar}(z) R_{21}^{\hbar}(-w)=N \phi\left(\frac{z-w}{N}, N \hbar\right)\left(r_{12}(z+N \hbar)-r_{12}(w+N \hbar)\right) \\
+N \phi\left(\frac{w-z}{N}, z\right) R_{12}^{\frac{z-w}{N}}(z+N \hbar)-N \phi\left(\frac{w-z}{N}, w\right) R_{12}^{\frac{z-w}{N}}(w+N \hbar) \\
+N^{2} 1 \otimes 1 \phi\left(\frac{z-w}{N}, N \hbar\right)\left(E_{1}(N \hbar)-E_{1}\left(N \hbar+\frac{z-w}{N}\right)\right),
\end{gathered}
$$

and

$$
\begin{gathered}
R_{12}^{\hbar}(z) R_{21}^{\hbar^{\prime}}(-z)=N \phi\left(\hbar^{\prime}-\hbar,-z\right)\left(r_{12}(z+N \hbar)-r_{12}\left(z+N \hbar^{\prime}\right)\right) \\
-N \phi\left(\hbar^{\prime}-\hbar, N \hbar\right) R_{12}^{\hbar-\hbar^{\prime}}(z+N \hbar)+N \phi\left(\hbar^{\prime}-\hbar, N \hbar^{\prime}\right) R_{12}^{\hbar-\hbar^{\prime}}\left(z+N \hbar^{\prime}\right) \\
+N^{2} 1 \otimes 1 \phi\left(\hbar^{\prime}-\hbar,-z\right)\left(E_{1}(z)-E_{1}\left(z+\hbar-\hbar^{\prime}\right)\right) .
\end{gathered}
$$

- Geometric interpretation. Due to the quasi-periodicities (1.38)-(1.41) the $R$-matrix have the following geometrical interpretation. Let $V_{1}\left(V_{2}\right)$ be a rank $N$ and degree one vector bundle over elliptic curve $\Sigma_{\tau}^{(1)}$ with coordinate $z_{1}\left(\Sigma_{\tau}^{(2)}\right.$ with coordinate $\left.z_{2}\right)$. Consider the bundle $V_{1} \otimes V_{2}$ over $\Sigma_{\tau}^{(1)} \times \Sigma_{\tau}^{(2)}$. Let $A u t_{\mathrm{PGL}(N)}\left(V_{1} \otimes V_{2}\right)$ be the automorphism group of the bundle (the gauge group). The sections $\Gamma\left(A u t_{\mathrm{PGL}(N)}\left(V_{1} \otimes V_{2}\right)\right)$ depends only on the anti-diagonal $\tilde{\Sigma}_{\tau}$ of $\Sigma_{\tau}^{(1)} \times \Sigma_{\tau}^{(2)}$ with the coordinate $z=z_{1}-z_{2}$. Let $\tilde{\Sigma}_{\tau}^{\prime}$ be the dual curve, $\hbar$ is the coordinate on $\tilde{\Sigma}_{\tau}^{\prime}$ and $\mathcal{P}$ is the Poincaré bundle $\mathcal{P}$ over $\tilde{\Sigma}_{\tau} \times \tilde{\Sigma}_{\tau}^{\prime}$ (1.5). Then the $R$-matrix (1.24) is a section

$$
R_{12}^{\hbar}(z) \in \Gamma\left(\left(A u t_{\mathrm{PGL}(N)}\left(V_{1} \otimes V_{2}\right)\right) \otimes \mathcal{P}\right) .
$$

- Green function. Similarly to (1.19) the $R$-matrix can be considered as the Green function of $\bar{\partial}$-operator:

$$
\bar{\partial} R_{12}^{\hbar}(z)=N P_{12} \delta^{2}(z, \bar{z}) .
$$

Properties (1.30)-(1.48) simply follows from their scalar counterparts except (1.33) which follows from the unitarity condition (1.27) in the classical limit (1.30). Identities for derivatives (1.49), (1.50) were obtained in [3, 15]. Degenerated Fay identities (1.53), (1.54) in $\operatorname{Mat}(N, \mathbb{C})^{\otimes 3}$ follows from the nondegenerated one (1.51) and local expansions (1.30), (1.34).

Our main interest (in this paper) is the Fay identity in $\operatorname{Mat}(N, \mathbb{C})^{\otimes 2}(1.52)$ and its degenerations (1.55), (1.56). We prove them below. The computational trick is based on the "arguments symmetry" property (1.29) and the scalar Fay identities (1.15)-(1.17).

Painlevé VI. As an application of the obtained formulae we construct higher-dimensional Lax pairs for the Painlevé VI equation. Denote the half-periods of the elliptic curve $\Sigma_{\tau}$ as

$$
\left\{\Omega_{a}, a=0,1,2,3\right\}=\left\{0, \frac{1}{2}, \frac{1+\tau}{2}, \frac{\tau}{2}\right\} .
$$

The Painlevé VI equation in the elliptic form [12] is

$$
\frac{d^{2} u}{d \tau^{2}}=-\sum_{a=0}^{3} \nu_{a}^{2} \wp^{\prime}\left(u+\Omega_{a}\right) .
$$


Let $N$ be an odd (positive) integer. Consider the following pair of block-matrices: 4 :

$$
\begin{gathered}
L(\hbar)=\frac{1}{2} \frac{d u}{d \tau}\left(\begin{array}{cc}
1 \otimes 1 & 0 \\
0 & -1 \otimes 1
\end{array}\right)+\sum_{a=0}^{3} \frac{\nu_{a}}{N \sqrt{-2}}\left(\begin{array}{cc}
0 & \mathcal{R}_{12}^{\hbar, a}(u) \\
\mathcal{R}_{21}^{\hbar, a}(-u) & 0
\end{array}\right) \\
M(\hbar)=\sum_{a=0}^{3} \frac{\nu_{a}}{N \sqrt{-2}}\left(\begin{array}{cc}
0 & \mathcal{F}_{12}^{\hbar, a}(u) \\
\mathcal{F}_{21}^{\hbar, a}(-u) & 0
\end{array}\right)
\end{gathered}
$$

where

$$
\begin{gathered}
\mathcal{R}_{12}^{\hbar, a}(u)=\exp \left(2 \pi \imath N \hbar \partial_{\tau} \Omega_{a}\right) R_{12}^{\hbar}\left(u+N \Omega_{a}\right), \\
\mathcal{R}_{21}^{\hbar, a}(-u)=\exp \left(-2 \pi \imath N \hbar \partial_{\tau} \Omega_{a}\right) R_{21}^{\hbar}\left(-u-N \Omega_{a}\right),
\end{gathered}
$$

and

$$
\begin{gathered}
\mathcal{F}_{12}^{\hbar, a}(u)=\exp \left(2 \pi \imath N \hbar \partial_{\tau} \Omega_{a}\right) F_{12}^{\hbar}\left(u+N \Omega_{a}\right), \\
\mathcal{F}_{21}^{\hbar, a}(-u)=\exp \left(-2 \pi \imath N \hbar \partial_{\tau} \Omega_{a}\right) F_{21}^{\hbar}\left(-u-N \Omega_{a}\right)
\end{gathered}
$$

with

$$
F_{a b}^{\hbar}(u)=\partial_{u} R_{a b}^{\hbar}(u) .
$$

The matrices $L(\hbar), M(\hbar) \in \operatorname{Mat}(2, \mathbb{C}) \otimes \operatorname{Mat}(N, \mathbb{C})^{\otimes 2}$. Their size equals $2 N^{2} \times 2 N^{2}$. The Painlevé VI equation (1.59) is equivalent to the monodromy preserving equation

$$
\frac{d}{d \tau} L(\hbar)-\left(\frac{1}{2 \pi \imath}\right) \frac{d}{d \hbar} M(\hbar)=[L(\hbar), M(\hbar)],
$$

where the Planck constant $\hbar$ plays the role of the spectral parameter (see [8]).

For $N=1$ the answer (1.60), (1.61) reproduces the elliptic $2 \times 2$ Lax pair proposed in [17].

The Lax pair (1.60), (1.61) works for even N's as well. But the Painlevé equation in this case has only one free constant:

$$
\frac{d^{2} u}{d \tau^{2}}=-\nu^{2} \wp^{\prime}(u), \quad \nu^{2}=\sum_{a=0}^{3} \nu_{a}^{2} .
$$

\section{Kronecker double series and Baxter-Belavin $R$-matrix}

Following idea suggested in [13] we derive here the Baxter-Belavin $R$-matrix as generalization of the Kronecker series.

$R$-matrix in Jacobi variables. Represent the elliptic curve $\Sigma_{\tau}(1.4)$ in the Jacobi form

$$
C_{q}=\mathbb{C} / q^{\mathbb{Z}}, \quad q=\mathbf{e}(\tau)=\exp 2 \pi \imath \tau .
$$

Consider the product $C_{q} \times C_{q}$ with the coordinates $s=\mathbf{e}(u), t=\mathbf{e}(z)$. Instead of the Kronecker function $\phi(z, u)$ we consider the distribution $g(s, t)$ on the space of the Laurent polynomials $\mathbb{C}\left[\left[s^{-1}, t^{-1}, s, t\right]\right]$. For $|q|<|t|<1$ it can be represented as the series

$$
g(s, t \mid q)=\sum_{n \in \mathbb{Z}} \frac{t^{n}}{q^{n} s-1} .
$$

\footnotetext{
${ }^{4}$ The coefficient $1 / \sqrt{-2}$ gives the normalization of the constants as in (1.59).
} 
If simultaneously $|q|<|s|<1$ then

$$
g(s, t \mid q)=-g^{+}(s, t \mid q)+g^{-}(s, t \mid q), \quad g^{+}(s, t \mid q)=\sum_{i, n \geq 0} s^{i} q^{i n} t^{n}, \quad g^{-}(s, t \mid q)=\sum_{i, n<0} s^{i} q^{i n} t^{n}
$$

or

$$
g(s, t \mid q)=1-\frac{1}{1-t}-\frac{1}{1-s}+g^{-}(s, t)-\sum_{i, n>0} s^{i} q^{i n} t^{n} .
$$

In the domain $|q|<|t|<1$ and $|q|<|s|<1$ we have

$$
\left.g(s, t \mid q)\right|_{s=\frac{1}{2 \pi \imath} \ln u, t=\frac{1}{2 \pi \imath} \ln z}=\phi(z, u) .
$$

The distribution $g(s, t \mid q)$ has the properties analogous to (1.6)-(1.9). In particular,

$$
g(s, t \mid q)=g(t, s \mid q) .
$$

It follows from (2.2) that

$$
g\left(s^{-1}, t^{-1} \mid q\right)=-g(s, t \mid q)+\delta(t)+\delta(s)-2,
$$

where $\delta(s)$ is the distribution on the space of the Laurent polynomials

$\mathbb{C}\left[t, t^{-1}\right]=\left\{\psi(t)=\sum_{l} c_{l} t^{l}\right\}$, defined by the functional $\langle\delta, \psi\rangle=\left.R e s\right|_{t=0} \psi(t)$ and represented by the formal series

$$
\delta(t)=\sum_{n \in \mathbb{Z}} t^{n}
$$

The analog of the quasiperiodic property (1.11) is the following. The distribution $g(s, t)$ is a solution of the difference equation on $t$ (the Green function) variable

$$
s g(s, t q \mid q)-g(s, t \mid q)=\delta(t)-1 .
$$

It defines the continuation of $g(s, t \mid q)$ from the annulus $|q|<|t|<1$ to $\mathbb{C}^{*}$. Due to (2.5) the similar equation can be written with respect to the $s$ variable.

Let $\eta=\mathbf{e}(\hbar)$. The $R$-matrix (1.24) takes the following form in variables $(s, t, \eta)$ :

$$
\begin{gathered}
R_{12}^{\hbar}(s)=\sum_{\alpha \in \mathbb{Z}_{N} \times \mathbb{Z}_{N}} s^{\alpha_{2} / N} g\left(s, \omega_{\alpha}+\hbar\right) T_{\alpha} \otimes T_{-\alpha}= \\
\sum_{\alpha \in \mathbb{Z}_{N} \times \mathbb{Z}_{N}} s^{\alpha_{2} / N}\left(\sum_{m, n} \mathbf{e}\left(n \alpha_{1} / N\right) q^{n\left(m+\alpha_{2} / N\right)} \eta^{n} s^{m}\right) T_{\alpha} \otimes T_{-\alpha} .
\end{gathered}
$$

It plays the role of the Green function for the difference operator

$$
\eta(\Lambda \otimes 1) R_{12}^{\hbar}(s q)\left(\Lambda^{-1} \otimes 1\right)-R_{12}^{\hbar}(s)=(\delta(s)-1) P_{12} .
$$

\section{Kronecker double series [16]}

The distribution $g(s, t \mid q)$ (and $\phi(z, u)$ ) can be represented as a Kronecker double series. Consider the lattice in $\mathbb{C}$

$$
W=\{\gamma=m+n \tau, m, n \in \mathbb{Z}\}
$$


Represent the argument $u$ of $\phi(z, u)$ as $u=u_{1}+u_{2} \tau\left(u_{1}, u_{2}\right.$ are real), and let

$$
\chi_{u}(\gamma)=\mathbf{e}\left(-m u_{2}+n u_{1}\right)
$$

be a character of the lattice $W\left(\chi_{u}(\gamma): W \rightarrow S^{1}\right)$, parameterized by $u \in \Sigma_{\tau}$. The Kronecker double series is defined as:

$$
S(z, u \mid \tau)=\sum_{\gamma \in W} \frac{\chi_{u}(\gamma)}{z+\gamma}
$$

From the definition we find that

$$
\begin{gathered}
S(z+1, u \mid \tau)=\mathbf{e}\left(u_{2}\right) S(z, u \mid \tau), \\
S(z+\tau, u \mid \tau)=\mathbf{e}\left(-u_{1}\right) S(z, u \mid \tau) .
\end{gathered}
$$

It was proved in [16] that $S(z, u \mid \tau)$ is related to the Kronecker function as

$$
S(z, u \mid \tau)=\mathbf{e}\left(u_{2} z\right) \phi(z, u)
$$

or in the Jacobi coordinates

$$
S(t, s \mid q)=t^{u_{2}} g(s, t \mid q) \text {. }
$$

Let us now pass to the $R$-matrix and describe it in terms of the Kronecker double series $S(z, u \mid \tau)$ (2.11).

Define the lattice $W$ by the two generators $\left(\alpha_{1} / N+\hbar_{1},\left(\alpha_{2} / N+\hbar_{2}\right) \tau\right)$, where $\hbar=\hbar_{1}+\hbar_{2} \tau$, $\hbar_{1,2} \in \mathbb{R}$. The corresponding character of $W$ is

$$
\chi_{(m, n)}(\alpha, \hbar)=\mathbf{e}\left(-m\left(\alpha_{2} / N+\hbar_{2}\right)+n\left(\alpha_{1} / N+\hbar_{1}\right)\right) .
$$

Then the $R$-matrix (1.24) is defined in terms of the Kronecker double series (2.11) as

$$
R_{12}^{\hbar}(z)=\mathbf{e}\left(-\hbar_{2} z\right) \sum_{(m, n) \in \mathbb{Z} \oplus \mathbb{Z}} \frac{\sum_{\alpha \in \mathbb{Z}_{N} \times \mathbb{Z}_{N}} \chi_{(m, n)}(\alpha, \hbar) T_{\alpha} \otimes T_{-\alpha}}{z+m+n \tau} .
$$

The quasi-periodicities (1.40), (1.41) now become evident. It follows from (2.13) that the singular behavior $z, \hbar \rightarrow 0$ of this representation is in agreement with (1.36).

We pass from $R_{12}^{\hbar}(z)$ to the modified matrix

$$
\tilde{R}_{12}^{\hbar}(z)=\mathbf{e}\left(\hbar_{2} z\right) R_{12}^{\hbar}(z) .
$$

It satisfies the Yang-Baxter equation and has the quasi-periodicities

$$
\begin{aligned}
& \tilde{R}_{12}^{\hbar}(z+1)=\mathbf{e}\left(\hbar_{2}\right)\left(Q^{-1} \otimes 1\right) \tilde{R}_{12}^{\hbar}(z)(Q \otimes 1), \\
& \tilde{R}_{12}^{\hbar}(z+\tau)=\mathbf{e}\left(\hbar_{1}\right)\left(\Lambda^{-1} \otimes 1\right) \tilde{R}_{12}^{\hbar}(z)(\Lambda \otimes 1),
\end{aligned}
$$

(compare with (1.40) $)$. In contrast with (1.41) $\tilde{R}$ is not holomorphic in $\hbar$ and is double-periodic. 
Remark 1 The representation (2.16) means that the elliptic $\tilde{R}$-matrix is represented as the averaging of the Yang matrix $z^{-1} P_{12}$ along the lattice $W$ twisted by the character (2.15).

From (1.30) we also find the representation for the classical $r$-matrix:

$$
r_{12}(z)=E_{1}(z) 1 \otimes 1+\sum_{m, n \in(\mathbb{Z} \oplus \mathbb{Z}) \backslash(0,0)} \frac{\sum_{\alpha \in \mathbb{Z}_{N} \times \mathbb{Z}_{N}} \chi_{(m, n)}(\alpha, 0) T_{\alpha} \otimes T_{-\alpha}}{z+m+n \tau}
$$

and

$$
m_{12}(z)=\frac{E_{1}^{2}(z)-\wp(z)}{2} 1 \otimes 1+\sum_{m, n \in(\mathbb{Z} \oplus \mathbb{Z}) \backslash(0,0)} \frac{\sum_{\alpha \in \mathbb{Z}_{N} \times \mathbb{Z}_{N}}(z+m+n \bar{\tau}) \chi_{(m, n)}(\alpha, 0) T_{\alpha} \otimes T_{-\alpha}}{(z+m+n \tau)(\bar{\tau}-\tau)} .
$$

\section{Derivation of identities}

Proposition 3.1 The R-matrix (1.24) satisfies the arguments symmetry property (1.29).

Proof: Using definitions (1.28) and (1.23) we have

$$
\begin{gathered}
R_{12}^{\frac{z}{N}}(N \hbar) P_{12}=\frac{1}{N} \sum_{\alpha, \beta} T_{\alpha} T_{\beta} \otimes T_{-\alpha} T_{-\beta} \varphi_{\alpha}\left(N \hbar, \omega_{\alpha}+\frac{z}{N}\right) \\
=\frac{1}{N} \sum_{\alpha, \beta} \kappa_{\alpha, \beta}^{2} T_{\alpha+\beta} \otimes T_{-\alpha-\beta} \varphi_{\alpha}\left(N \hbar, \omega_{\alpha}+\frac{z}{N}\right) .
\end{gathered}
$$

Since $\kappa_{\alpha, \beta}=\kappa_{\alpha, \alpha+\beta}$, the property (1.29) is equivalent to the following set of $N^{2}$ identities:

$$
\frac{1}{N} \sum_{\alpha} \kappa_{\alpha, \gamma}^{2} \varphi_{\alpha}\left(N \hbar, \omega_{\alpha}+\frac{z}{N}\right)=\varphi_{\gamma}\left(z, \omega_{\gamma}+\hbar\right), \quad \forall \gamma \in \mathbb{Z}^{\times 2}
$$

or

$$
\frac{1}{N} \sum_{\alpha} \kappa_{\alpha, \gamma}^{2} \varphi_{\alpha}\left(z, \omega_{\alpha}+\hbar\right)=\varphi_{\gamma}\left(N \hbar, \omega_{\gamma}+\frac{z}{N}\right), \quad \forall \gamma \in \mathbb{Z}^{\times 2} .
$$

The latter is verified by comparing residues. To do it we also need the relation for the sums of $N$-th roots of 1 (it also follows from $P_{12}^{2}=1$ ):

$$
\sum_{\alpha} \kappa_{\alpha, \gamma}^{2}=N^{2} \delta_{\gamma, 0}
$$

Let us calculate the residue of both parts of (3.2) at $\hbar=-\omega_{\mu}$. The answer for the r.h.s. is obviously $\delta_{\mu, \gamma} \exp \left(2 \pi \imath \partial_{\tau} \omega_{\gamma} z\right)$ due to (1.8). For the l.h.s. we have:

$$
\begin{gathered}
\operatorname{Res}_{\hbar=-\omega_{\mu}} \frac{1}{N} \sum_{\alpha} \kappa_{\alpha, \gamma}^{2} \varphi_{\alpha}\left(N \hbar, \omega_{\alpha}+\frac{z}{N}\right)=\operatorname{Res}_{\hbar=0} \frac{1}{N} \sum_{\alpha} \kappa_{\alpha, \gamma}^{2} \varphi_{\alpha}\left(N \hbar-N \omega_{\mu}, \omega_{\alpha}+\frac{z}{N}\right) \\
\stackrel{1.10,(1.11)}{=} \operatorname{Res}_{\hbar=0} \frac{1}{N} \sum_{\alpha} \kappa_{\alpha, \gamma}^{2} \kappa_{\alpha,-\mu}^{2} \exp \left(2 \pi \imath \partial_{\tau} \omega_{\mu} z\right) \varphi_{\alpha}\left(N \hbar, \omega_{\alpha}+\frac{z}{N}\right) \\
\stackrel{1.8}{=} \frac{1}{N} \sum_{\alpha} \kappa_{\alpha, \gamma-\mu}^{2} \exp \left(2 \pi \imath \partial_{\tau} \omega_{\mu} z\right) \frac{1}{N} \stackrel{3.4}{=} \delta_{\mu, \gamma} \exp \left(2 \pi \imath \partial_{\tau} \omega_{\mu} z\right) .
\end{gathered}
$$


Proposition 3.2 The R-matrix (1.24) satisfies the Fay identity (1.52) in $\operatorname{Mat}(N, \mathbb{C})^{\otimes 2}$.

Proof: Consider

$$
R_{12}^{\hbar}(z) R_{21}^{\hbar^{\prime}}(-w)=-\sum_{\alpha, \beta} \kappa_{\alpha, \beta}^{2} T_{\alpha+\beta} \otimes T_{-\alpha-\beta} \varphi_{\alpha}\left(z, \omega_{\alpha}+\hbar\right) \varphi_{\beta}\left(w, \omega_{\beta}-\hbar^{\prime}\right)=
$$

Here we already used $R_{21}^{\hbar^{\prime}}(-w)=-R_{12}^{-\hbar^{\prime}}(w)$. Apply the Fay identity (1.15), then (3.3) , and then (1.15) again:

$$
\begin{gathered}
=-\sum_{\alpha, \beta} \kappa_{\alpha, \beta}^{2} T_{\alpha+\beta} \otimes T_{-\alpha-\beta} \varphi_{\alpha}\left(z-w, \omega_{\alpha}+\hbar\right) \varphi_{\alpha+\beta}\left(w, \omega_{\alpha+\beta}+\hbar-\hbar^{\prime}\right) \\
-\sum_{\alpha, \beta} \kappa_{\alpha, \beta}^{2} T_{\alpha+\beta} \otimes T_{-\alpha-\beta} \varphi_{\beta}\left(w-z, \omega_{\beta}-\hbar^{\prime}\right) \varphi_{\alpha+\beta}\left(z, \omega_{\alpha+\beta}+\hbar-\hbar^{\prime}\right) \\
=-N \sum_{\gamma} T_{\gamma} \otimes T_{-\gamma} \varphi_{\gamma}\left(N \hbar, \omega_{\gamma}+\frac{z-w}{N}\right) \varphi_{\gamma}\left(w, \omega_{\gamma}+\hbar-\hbar^{\prime}\right) \\
+N \sum_{\gamma} T_{\gamma} \otimes T_{-\gamma} \varphi_{\gamma}\left(N \hbar^{\prime}, \omega_{\gamma}+\frac{z-w}{N}\right) \varphi_{\gamma}\left(z, \omega_{\gamma}+\hbar-\hbar^{\prime}\right) \\
=N \sum_{\gamma} T_{\gamma} \otimes T_{-\gamma}\left(-\phi\left(N \hbar, \frac{z-w}{N}+\hbar^{\prime}-\hbar\right) \varphi_{\gamma}\left(w+N \hbar, \omega_{\gamma}+\hbar-\hbar^{\prime}\right)\right. \\
\quad-\phi\left(w, \hbar-\hbar^{\prime}-\frac{z-w}{N}\right) \varphi_{\gamma}\left(w+N \hbar, \omega_{\gamma}+\frac{z-w}{N}\right) \\
+\phi\left(N \hbar^{\prime}, \frac{z-w}{N}+\hbar^{\prime}-\hbar\right) \varphi_{\gamma}\left(z+N \hbar^{\prime}, \omega_{\gamma}+\hbar-\hbar^{\prime}\right) \\
\left.+\phi\left(z, \hbar-\hbar^{\prime}-\frac{z-w}{N}\right) \varphi_{\gamma}\left(z+N \hbar^{\prime}, \omega_{\gamma}+\frac{z-w}{N}\right)\right) .
\end{gathered}
$$

Proposition 3.3 The R-matrices (1.24) and (1.31) satisfies the degenerated Fay identities (1.55), (1.56) in $\operatorname{Mat}(N, \mathbb{C})^{\otimes 2}$.

Proof: We begin with (1.55). Consider

$$
R_{12}^{\hbar}(z) R_{21}^{\hbar}(-w)=-\sum_{\alpha, \beta} \kappa_{\alpha, \beta}^{2} T_{\alpha+\beta} \otimes T_{-\alpha-\beta} \varphi_{\alpha}\left(z, \omega_{\alpha}+\hbar\right) \varphi_{\beta}\left(w, \omega_{\beta}-\hbar\right) .
$$

Subdivide it into two parts: $\sum_{\alpha, \beta}=\sum_{\alpha \neq-\beta}+\sum_{\alpha=-\beta}$. The first part is transformed as in the previous Proposition (via (1.15), then (3.3), and then (1.15) again)

$$
\begin{gathered}
\sum_{\alpha \neq-\beta}=-\sum_{\alpha \neq-\beta} \kappa_{\alpha, \beta}^{2} T_{\alpha+\beta} \otimes T_{-\alpha-\beta} \varphi_{\alpha}\left(z-w, \omega_{\alpha}+\hbar\right) \varphi_{\alpha+\beta}\left(w, \omega_{\alpha+\beta}\right) \\
-\sum_{\alpha \neq-\beta} \kappa_{\alpha, \beta}^{2} T_{\alpha+\beta} \otimes T_{-\alpha-\beta} \varphi_{\beta}\left(w-z, \omega_{\beta}-\hbar\right) \varphi_{\alpha+\beta}\left(z, \omega_{\alpha+\beta}\right)
\end{gathered}
$$




$$
\begin{gathered}
=\ldots=-N \phi\left(\frac{z-w}{N}, N \hbar\right) \sum_{\gamma \neq 0} T_{\gamma} \otimes T_{-\gamma} \varphi_{\gamma}\left(w+N \hbar, \omega_{\gamma}\right) \\
-N \phi\left(\frac{w-z}{N}, w\right) \sum_{\gamma \neq 0} T_{\gamma} \otimes T_{-\gamma} \varphi_{\gamma}\left(w+N \hbar, \omega_{\gamma}+\frac{z-w}{N}\right) \\
+N \phi\left(\frac{z-w}{N}, N \hbar\right) \sum_{\gamma \neq 0} T_{\gamma} \otimes T_{-\gamma} \varphi_{\gamma}\left(z+N \hbar, \omega_{\gamma}\right) \\
+N \phi\left(\frac{w-z}{N}, z\right) \sum_{\gamma \neq 0} T_{\gamma} \otimes T_{-\gamma} \varphi_{\gamma}\left(z+N \hbar, \omega_{\gamma}+\frac{z-w}{N}\right)
\end{gathered}
$$

By adding (and subtracting) scalar terms $(1 \otimes 1)$ to each line one obtains the first and the second lines of (1.55). The input to the scalar part should be summed up together with

$$
\begin{gathered}
\sum_{\alpha=-\beta}=1 \otimes 1 \sum_{\alpha} \varphi_{\alpha}\left(z, \omega_{\alpha}+\hbar\right) \varphi_{\alpha}\left(-w, \omega_{\alpha}+\hbar\right) \\
\stackrel{\text { 1.17] }}{=} 1 \otimes 1 \sum_{\alpha} \varphi_{\alpha}\left(z-w, \omega_{\alpha}+\hbar\right)\left(E_{1}(z)-E_{1}(w)+E_{1}\left(\hbar+\omega_{\alpha}\right)-E_{1}\left(z-w+\hbar+\omega_{\alpha}\right)\right) .
\end{gathered}
$$

The latter expression is transformed via (3.3) for $\gamma=0$

$$
\sum_{\alpha} \varphi_{\alpha}\left(z-w, \omega_{\alpha}+\hbar\right)=N \phi\left(N \hbar, \frac{z-w}{N}\right)
$$

and its derivative (1.13), (1.14) with respect to $\hbar$ :

$$
\begin{gathered}
\sum_{\alpha} \varphi_{\alpha}\left(z-w, \omega_{\alpha}+\hbar\right)\left(E_{1}\left(z-w+\hbar+\omega_{\alpha}\right)-E_{1}\left(\hbar+\omega_{\alpha}\right)\right) \\
=N^{2} \phi\left(N \hbar, \frac{z-w}{N}\right)\left(E_{1}\left(N \hbar+\frac{z-w}{N}\right)-E_{1}(N \hbar)\right) .
\end{gathered}
$$

This finishes the proof of (1.55). The identity (1.56) can be derived similarly. Equivalently, (1.56) follows from (1.55) by using the properties (1.29) and (1.37).

\section{Higher-dimensional elliptic Lax pairs for Painlevé VI}

Different types of matrix-valued Lax pairs for Painlevé equations are known (see e.g. [7, 5, 10]). In this section we construct $R$-matrix valued generalization of the elliptic $2 \times 2$ Lax pair suggested in [17].

Proposition 4.1 The Painlevé VI equation in the elliptic form (1.59) is equivalent to the monodromy preserving equation (1.65) with the Lax pair (1.60)-(1.64) and the elliptic R-matrix (1.24) for odd $N$.

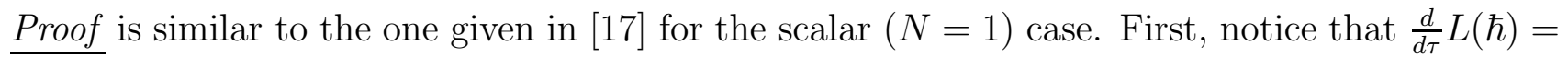
$\frac{\overline{d u} \partial_{u} L}{d \tau}(\hbar)+\partial_{\tau} L(\hbar)$, where the last term is the derivative by explicit dependence on $\tau$. It is canceled out by $\frac{1}{2 \pi \imath} \frac{d}{d \hbar} M(\hbar)$ due to the heat equation (1.48) $2 \pi \imath \partial_{\tau} \mathcal{R}_{b c}^{\hbar, a}(u)=\partial_{\hbar} \mathcal{F}_{b c}^{\hbar, a}(u)$. 
Denote

$$
L^{a}=\left(\begin{array}{cc}
0 & \mathcal{R}_{12}^{\hbar, a}(u) \\
\mathcal{R}_{21}^{\hbar, a}(-u) & 0
\end{array}\right), \quad M^{a}=\left(\begin{array}{cc}
0 & \mathcal{F}_{12}^{\hbar, a}(u) \\
\mathcal{F}_{21}^{\hbar, a}(-u) & 0
\end{array}\right)
$$

The main statement which we need to verify is that for $a \neq b$

$$
\left[L^{a}, M^{b}\right]+\left[L^{b}, M^{a}\right]=0
$$

i.e. the input to $[L(\hbar), M(\hbar)]$ comes only from $\left[L^{a}, M^{a}\right]$. Indeed, it follows from the unitarity condition (1.27) that

$$
\mathcal{R}_{12}^{\hbar, a}(u) \mathcal{R}_{21}^{\hbar, a}(-u)=R_{12}^{\hbar}\left(u+N \Omega_{a}\right) R_{21}^{\hbar}\left(-u-N \Omega_{a}\right)=N^{2}\left(\wp(N \hbar)-\wp\left(u+N \Omega_{a}\right)\right) .
$$

Differentiating (4.3) with respect to $u$ we get

$$
\mathcal{F}_{12}^{\hbar, a}(u) \mathcal{R}_{21}^{\hbar, a}(-u)-\mathcal{R}_{12}^{\hbar, a}(u) \mathcal{F}_{21}^{\hbar, a}(-u)=-N^{2} \wp^{\prime}\left(u+N \Omega_{a}\right) .
$$

This identity provides the equation of motion. Notice that in order to have all four constants $N$ should be odd since $\wp^{\prime}\left(u+N \Omega_{a}\right)=\wp^{\prime}\left(u+\Omega_{a}\right)$ in this case. If $N$ is even then $\wp^{\prime}\left(u+N \Omega_{a}\right)=\wp^{\prime}(u)$, and we have only one constant as in (1.66) .

To prove (4.2) let us recall that in the scalar case this followed from

$$
\begin{gathered}
\varphi_{a}\left(\hbar, u+\Omega_{a}\right) f_{b}\left(\hbar,-u-\Omega_{b}\right)-f_{b}\left(\hbar, u+\Omega_{b}\right) \varphi_{a}\left(\hbar,-u-\Omega_{a}\right) \\
\varphi_{b}\left(\hbar, u+\Omega_{b}\right) f_{a}\left(\hbar,-u-\Omega_{a}\right)-f_{a}\left(\hbar, u+\Omega_{a}\right) \varphi_{b}\left(\hbar,-u-\Omega_{b}\right)=0,
\end{gathered}
$$

where

$$
f_{a}\left(z, u+\Omega_{a}\right)=\left.\exp \left(2 \pi \imath \partial_{\tau} \Omega_{a} \hbar\right) \partial_{w} \phi(\hbar, w)\right|_{w=u+\Omega_{a}}
$$

is the scalar analogue of $\mathcal{F}_{12}^{\hbar, a}(u)$. The identity (4.5) appears from (1.16) and (1.10)-(1.11) as follows:

$$
\begin{gathered}
\varphi_{a}\left(\hbar, u+\Omega_{a}\right) \varphi_{b}\left(\hbar,-u-\Omega_{b}\right)+\varphi_{b}\left(\hbar, u+\Omega_{b}\right) \varphi_{a}\left(\hbar,-u-\Omega_{a}\right)= \\
\varphi_{a+b}\left(\hbar, \Omega_{a}+\Omega_{b}\right)\left(2 E_{1}(\hbar)-E_{1}\left(\hbar+\Omega_{a}-\Omega_{b}\right)-E_{1}\left(\hbar+\Omega_{b}-\Omega_{a}\right)\right) .
\end{gathered}
$$

The r.h.s. of (4.6) is independent of $u$. The derivative of (4.6) with respect to $u$ gives (4.5).

Similarly to (4.6) it follows from the degenerated Fay identity (1.55) that

$$
\begin{gathered}
\mathcal{R}_{12}^{\hbar, a}(u) \mathcal{R}_{21}^{\hbar, b}(-u)+\mathcal{R}_{12}^{\hbar, b}(u) \mathcal{R}_{21}^{\hbar, a}(-u) \\
=N^{2} 1 \otimes 1 \varphi_{a+b}\left(N \hbar, \Omega_{a}+\Omega_{b}\right)\left(2 E_{1}(N \hbar)-E_{1}\left(N \hbar+\Omega_{a}-\Omega_{b}\right)-E_{1}\left(N \hbar+\Omega_{b}-\Omega_{a}\right)\right) .
\end{gathered}
$$

It can be verified directly using (1.10)-(1.11) which can be re-written as

$$
\phi\left(z, w+\Omega_{a}\right)=\exp \left(-2 \pi \imath z \partial_{\tau} \Omega_{a}\right) \phi\left(z, w-\Omega_{a}\right) .
$$

The r.h.s. of (4.7) is scalar and independent of $u$. The derivative of (4.7) with respect to $u$ gives

$$
\mathcal{F}_{12}^{\hbar, a}(u) \mathcal{R}_{21}^{\hbar, b}(-u)-\mathcal{R}_{12}^{\hbar, a}(u) \mathcal{F}_{21}^{\hbar, b}(-u)+\mathcal{F}_{12}^{\hbar, b}(u) \mathcal{R}_{21}^{\hbar, a}(-u)-\mathcal{R}_{12}^{\hbar, b}(u) \mathcal{F}_{21}^{\hbar, a}(-u)=0 .
$$

This identity underlies (4.2). 


\section{Acknowledgments}

We would like to thank N. Slavnov and A. Zabrodin for useful remarks. The work was done at the

Steklov Mathematical Institute RAS, Moscow. It was supported by RSCF grant 14-50-00005.

\section{References}

[1] M. Aguiar, Contemporary Mathematics 267 (2000) 1-29.

[2] R.J. Baxter, Ann. Phys. 70 (1972) 193-228.

[3] V.V. Bazhanov, Y.G. Stroganov, Proc. VI-th. Int. Seminar on High Energy Physics and Quantum Field Theory (1983) 52-54.

[4] A.A. Belavin, Nucl. Phys. B, 180 (1981) 189-200.

A.A. Belavin, V.G. Drinfeld, Funct. Anal. Appl. 16 (1982) 159-180.

[5] B. Dubrovin, M. Mazzocco, Invent. Math. 141 (2000) 55-147; arXiv:math/9806056 [math.AG].

P. Boalch, Proc. of the London Math. Soc., 90 (2005) 167-208.

[6] J.D. Fay, Lecture Notes in Mathematics, Vol. 352 (1973) Berlin: Springer.

[7] M. Jimbo, T. Miwa, Physica D, 2 (1981) 407-448.

N. Joshi, A. Kitaev, P. Treharne, J. Math. Phys. 48 (2007) 103512; arXiv:0706.1750.

M. Noumi, Y. Yamada, Microlocal analysis and complex Fourier analysis, (2002) 238252; arXiv:math-ph/0203029

G. Aminov, S. Arthamonov, arXiv:1112.4688 [nlin.SI].

[8] A. Levin, M. Olshanetsky, A. Zotov, JHEP 10 (2014) 109; arXiv:1408.6246 [hep-th].

[9] A. Levin, M. Olshanetsky, A. Zotov, JHEP 07 (2014) 012; arXiv:1405.7523 [hep-th].

A. Levin, M. Olshanetsky, A. Zotov, Nuclear Physics B, 887 (2014) 400-422; arXiv:1406.2995 [math-ph].

[10] A. Levin, M. Olshanetsky, Calogero-Moser-Sutherlend models (Montreal, 1997), CRM Ser. Math. Phys., Springer (2000) 313-332, arXiv: alg-geom/9706010

A. Levin, M. Olshanetsky, A. Zotov, Commun. Math. Phys. Vol. 268 (2006) 67-103; arXiv: math/0508058 [math.QA];

A. Levin, A. Zotov, AMS Transl. (2), 221 (2007) 173-184.

A. Zabrodin, A. Zotov, J. Math. Phys. 53 (2012) 073508; arXiv:1107.5672 [math-ph].

A. Zabrodin, A. Zotov, Construct. Approx., Painlevé Equations Special Issue II (2015) to appear; DOI:10.1007/s00365-015-9284-4; arXiv:1212.5813 [math-ph].

A. Levin, M. Olshanetsky, A. Zotov, Russ. Math. Surveys 69:1 (2014) 35-118; arXiv:1311.4498 [math-ph].

[11] D. Mumford, Tata Lectures on Theta I, II, Birkhäuser Boston, 1983, 1984.

[12] P. Painlevé, C. R. Acad. Sci. (Paris) 143 (1906) 1111-1117.

Yu. Manin, AMS Transl. (2), 186 (1998) 131-151.

[13] A. Polishchuk, Advances in Mathematics 168:1 (2002) 56-95.

[14] M.P. Richey, C.A. Tracy, Journal of Statistical Physics, 42 (1986) 311-348.

K. Hasegawa, J. Math. Phys. 35: 11 (1994) 6158-6171.

[15] L.A. Takhtajan, Zapiski nauch. seminarov LOMI, 133 (1984) 258-276 (in Russian).

[16] A. Weil, Elliptic functions according to Eisenstein and Kronecker, Springer-Verlag, Berlin-Heidelberg-New York, (1976).

[17] A. Zotov, Lett. Math. Phys. 67 (2004) 153-165, arXiv:hep-th/0310260. 